\title{
CONHECIMENTOS DE PROFESSORES SOBRE PERDA AUDITIVA E SUAS ATITUDES FRENTE À INCLUSÃO
}

\author{
Teachers' knowledge about hearing loss and attitudes \\ towards the inclusion
}

\author{
Eliane Maria Carrit Delgado-Pinheiro ${ }^{(1)}$, Sadao Omote ${ }^{(2)}$
}

\begin{abstract}
RESUMO
Objetivos: verificar os conhecimentos dos professores sobre a perda auditiva, suas opiniões sobre a educação de alunos com esse tipo de privação sensorial e também conhecer suas atitudes frente à proposta da inclusão. Métodos: participaram desta pesquisa quatro grupos de professores do ensino fundamental, com e sem experiência com aluno com perda auditiva. Os instrumentos utilizados na coleta de dados foram a Escala Lickert de Atitudes Sociais em Relação à Inclusão (ELASI) e um questionário. Comparações entre os resultados de diferentes grupos, por meio de provas estatísticas apropriadas, foram feitas, sempre que a natureza dos dados o recomendava. Resultados: os professores de $1^{\text {a }}$ a $4^{\underline{a}}$ séries, com e sem experiência com alunos com perda auditiva, apresentaram respostas semelhantes com referência às atitudes sociais acerca da inclusão, tanto na dimensão ideológica quanto na operacional. Professores de $5^{\underline{a}}$ a $8^{\underline{a}}$ séries, com e sem experiência com alunos com perda auditiva, apresentaram respostas semelhantes nos itens ideológicos, porém divergiram nos itens operacionais. Em relação aos conhecimentos, os grupos de professores com experiência não apresentaram conhecimentos diferenciados sobre aspectos relativos à perda auditiva, quando comparados com os grupos de professores sem experiência, e todos os grupos enfatizaram os aspectos comunicativos. Conclusão: a análise revela que os dados provenientes de diferentes instrumentos se complementam e sugerem que os professores são ideologicamente favoráveis à inclusão, entretanto, não têm conhecimentos suficientes para operacionalizar tal proposta.
\end{abstract}

DESCRITORES: Educação; Reabilitação de Deficientes Auditivos; Surdez; Perda Auditiva

\section{INTRODUÇÃO}

Os avanços tecnológicos, tanto no diagnóstico precoce da deficiência auditiva, quanto nos recursos de acesso aos sons da fala, como os aparelhos de amplificação sonora individuais digitais, sistema de frequência modulada e implante coclear ${ }^{1-6}$, além do debate da utilização da LIBRAS

(1) Fonoaudióloga; Professora Assistente Doutora do Departamento de Fonoaudiologia da Universidade Estadual Paulista, UNESP, Marília, SP, Brasil; Doutora em Educação pela Universidade Estadual Paulista, Campus de Marília.

(2) Psicólogo; Professor Titular do Departamento de Educação Especial da Universidade Estadual Paulista, UNESP, Marília, SP, Brasil; Doutor em Psicologia (Psicologia Experimental) pela Universidade de São Paulo e Livre-docência em Educação Especial pela Universidade Estadual Paulista.

Conflito de interesses: inexistente
(Língua Brasileira de Sinais) ${ }^{7}$ e diferentes estratégias de comunicação de acordo com a necessidade do aluno deficiente auditivo, são exemplos de variáveis que permeiam a inclusão do aluno deficiente auditivo no ensino regular. É salutar que profissionais da saúde e educação acompanhem conjuntamente essa temática.

Os conhecimentos que os professores possuem sobre a deficiência auditiva, para exercer sua prática profissional, e suas atitudes frente ao processo de inclusão são pontos determinantes para o sucesso dessa proposta ${ }^{8-11}$.

Diversos estudos procuram avaliar as atitudes sociais de professores envolvidos com alunos com necessidades especiais no ensino regular. Alguns estudos enfocam a proposta da integração, enquanto outros abordam a inclusão, porém, independente da proposta educacional em questão, o estudo das atitudes é ressaltado para que se 
viabilizem as alterações no sistema educacional ${ }^{12-14}$. O fato de conhecer as características, necessidades e habilidades de alunos com necessidades educacionais especiais poderão influenciar positivamente, para que as pessoas tenham atitudes favoráveis em relação à inclusão ${ }^{15}$.

No Brasil, pesquisas têm evidenciado que os profissionais envolvidos com a área de Educação apresentam opinião relativamente favorável em relação à perspectiva de alunos com necessidades educacionais especiais estudarem em classes comuns ${ }^{16,17}$. Um ponto frequentemente apontado nas discussões dos estudos são as dificuldades encontradas para concretizar esse trabalho, como falta de estrutura, de conhecimentos suficientes e número excessivo de alunos nas salas de aula.

O objetivo do presente estudo, realizado com professores com e sem experiência com alunos com perda auditiva, foi o de verificar os conhecimentos desses profissionais sobre essa privação sensorial, as suas opiniões sobre a educação desses alunos e também as atitudes desses profissionais frente à proposta de inclusão.

\section{MÉTODOS}

Nesse estudo foi utilizada a Escala Lickert de Atitudes Sociais em relação à Inclusão (ELASI). A ELASI é composta por duas formas equivalentes (A e B). A elaboração de duas escalas equivalentes justifica-se pela aplicabilidade em estudos que necessitem de duas avaliações similares, por exemplo, antes e após um programa de intervenção. As formas A e B da ELASI foram aplicadas nessa pesquisa, pois permitia iniciar a utilização desse instrumento e posteriormente verificar a equivalência dos resultados.

Os itens da escala são enunciados que abordam temas relacionados à inclusão. Cada item é seguido de 5 alternativas que expressam o grau de concordância ou discordância, em relação ao conteúdo do enunciado. Essas alternativas são: concordo inteiramente, concordo mais ou menos, nem discordo nem concordo, discordo mais ou menos e discordo inteiramente.

Assim, cada forma da escala contém 30 itens que correspondem à escala de atitudes sociais em relação à inclusão. Metade dos itens tem enunciados favoráveis à inclusão e a outra metade, desfavoráveis.

As formas A e B da ELASI contêm enunciados com dimensões ideológica e operacional. Os enunciados referentes à dimensão ideológica contemplam princípios que fundamentam a proposta da inclusão, enquanto que os enunciados referentes à dimensão operacional abordam as ações para se colocar em prática os princípios da inclusão.

$\mathrm{Na}$ forma $\mathrm{A}$, a dimensão ideológica é composta por 21 enunciados, dos quais 11 são favoráveis e 10 desfavoráveis em relação à inclusão. Com respeito à dimensão operacional, é formada por 9 enunciados, sendo 4 favoráveis e 5 desfavoráveis à inclusão. Na forma $\mathrm{B}$, a dimensão ideológica é composta por 19 enunciados, dos quais 10 são favoráveis e 9 desfavoráveis à inclusão. Com relação à dimensão operacional, esta é formada por 11 enunciados, sendo 5 favoráveis e 6 desfavoráveis à inclusão. Alguns exemplos de enunciados ideológicos e operacionais, tanto favoráveis quanto desfavoráveis, são apresentados a seguir:

- Itens Ideológicos:

Favoráveis: "Devemos aceitar e conviver com a singularidade das pessoas".

Desfavoráveis: "Não há benefícios com a inclusão, ela prejudica tanto alunos deficientes quanto normais".

- Itens Operacionais:

Favoráveis: "O currículo deve ser adaptado para garantir ao aluno deficiente a sua participação em classe comum".

Desfavoráveis: "O melhor local de atendimento educacional para o deficiente é na instituição especializada".

A ELASI foi construída obedecendo a todos os requisitos necessários para a sua padronização e confiabilidade ${ }^{14,18}$.

Nos itens favoráveis, o assinalamento da alternativa "a", que expressa concordância total com o enunciado, recebeu a nota 5; a alternativa "b", a nota 4 e assim por diante, até a alternativa "e", que expressa discordância total, a nota 1. Nos itens desfavoráveis, os valores das notas foram invertidos, isto é, nota 1 para alternativa "a", até a nota 5, para alternativa "e".

O escore total de cada professor foi calculado somando-se as notas obtidas em cada um dos 30 itens da escala. Esse escore total de cada professor pode variar de 30 a 150.

O grupo de professores com experiência com aluno deficiente auditivo foi comparado com o de professores sem essa experiência, usando esses escores de atitudes sociais em relação à inclusão. Essa comparação foi feita para os professores de $1^{\mathrm{a}}$ a $4^{\mathrm{a}}$ séries e de $5^{\mathrm{a}}$ a $8^{\mathrm{a}}$ séries, separadamente.

Foi também realizada uma separação dos itens ideológicos e operacionais da ELASI, e os grupos de professores com e sem experiência com alunos deficientes auditivos foram comparados em relação a essas duas dimensões. Tendo em vista a 
variação no número de enunciados, para cada uma dessas dimensões, cabe destacar que os escores da dimensão ideológica variaram de 21 a 105, na Forma A, e de 19 a 95, na Forma B, enquanto os escores da dimensão operacional variaram de 9 a 45, na Forma A, e de 11 a 45, na Forma B.

Além da aplicação da ELASI, foi também utilizado um questionário aberto para verificar os conhecimentos sobre a perda auditiva, opiniões sobre a educação do aluno com perda auditiva e os relatos das experiências dos professores. O questionário continha 16 questões, com perguntas relacionadas aos conhecimentos dos professores sobre os conceitos de perda auditiva, recursos para o aproveitamento da audição residual, graus de perda auditiva e estratégias utilizadas para comunicação com o aluno deficiente auditivo e qual a melhor forma de ensino para esse aluno.

Foi feita a validação semântica do questionário, preliminarmente junto a duas especialistas. Foi também aplicado a um grupo de 29 professores do ensino regular, pertencentes às escolas diferentes daquelas nas quais o presente estudo foi realizado.

Os participantes deste estudo foram 72 professores do ensino regular, divididos em quatro grupos: 26 professores de $1^{\underline{a}}$ a $4^{\underline{a}}$ séries que nunca tiveram contato, no ambiente de sala de aulas, com crianças deficientes auditivas, 19 professores de $1^{\text {a }}$ a $4^{\mathrm{a}}$ séries que atuaram ou atuam com crianças deficientes auditivas, no ensino regular, 7 professores de $5^{\underline{a}}$ a $8^{\underline{a}}$ séries que nunca tiveram contato, no ambiente de sala de aula, com crianças deficientes auditivas e 20 professores de $5^{\underline{a}}$ a $8^{\underline{a}}$ séries que atuam com crianças deficientes auditivas, no ensino regular.

Para se determinar à amostra de professores, primeiramente obteve-se, junto à Diretoria de Ensino Estadual e à Secretaria Municipal de Educação, uma lista com nome e telefone de escolas de ensino público estadual e municipal.

Após a realização do levantamento dessas informações, foi feita a seleção das escolas.

A escola selecionada para constituir a amostra de professores do grupo 1 , (professores de $1^{\underline{a}}$ a $4^{a}$ séries, sem experiência), foi aquela que continha maior número de classes, na rede municipal de ensino.

Tendo em vista que, nessa escola, havia duas crianças deficientes auditivas, os professores dessas crianças, bem como aqueles que já haviam atuado com esses alunos, fizeram parte do segundo grupo.

De acordo com os registros, nas Redes Municipal e Estadual de Ensino, 9 crianças surdas estavam inseridas no ensino regular de $1^{\underline{a}}$ a $4^{\underline{a}}$ séries. Os professores e ex-professores dessas crianças participaram deste estudo. Assim, o segundo grupo foi composto por esses professores que estavam atuando com alunos deficientes auditivos inseridos no ensino fundamental e somaram-se a essa amostra os professores que já haviam atuado com crianças com perda auditiva no ensino regular.

Em relação ao ensino fundamental de $5^{\mathrm{a}}$ a $8^{\mathrm{a}}$ séries, 12 alunos deficientes auditivos estavam inseridos nessas séries.

Duas escolas, que tinham alunos com perda auditiva incluídos no ensino fundamental de $5^{\underline{a}}$ a $8^{\text {a }}$ séries, foram sorteadas para compor o grupo de professores que atuavam com alunos deficientes auditivos nessas séries. Em cada escola, dois alunos com perda auditiva frequentavam o ensino regular. Os professores de todas as disciplinas participaram deste estudo, entre os quais alguns que lecionavam para os dois alunos.

A amostra de professores de $5^{\mathrm{a}}$ a $8^{\mathrm{a}}$ séries que não tinham experiência com alunos com deficiência auditiva foi proveniente de uma das escolas sorteadas anteriormente.

Todos os sujeitos participantes assinaram o "termo de Consentimento Livre e Esclarecido".

Para a coleta dos dados foi aplicado a ELASI junto aos professores participantes deste estudo e utilizado um questionário aberto para verificar os conhecimentos sobre a perda auditiva, opiniões sobre a educação do aluno com perda auditiva e os relatos das experiências dos professores.

Para a análise dos dados tanto da ELASI, como do questionário, os professores da $1^{\underline{a}}$ a $4^{\underline{a}}$ série constituíram um grupo e os de $5^{\underline{a}}$ a $8^{\underline{a}}$, outro grupo. Estes grupos foram subdivididos com relação à experiência ou não com alunos deficientes. O grupo de $5^{\mathrm{a}}$ a $8^{\mathrm{a}}$ série respondeu apenas a forma $A$ da ELASI, pois foi constituído por um grupo menor de sujeitos.

$\mathrm{Na}$ ELASI, estes grupos foram analisados com relação aos escores obtidos nos itens das dimensões ideológicas e operacionais.

O estudo foi submetido ao Comitê de Ética em Pesquisa da Faculdade de Filosofia e Ciências da Universidade Estadual Paulista - FFC/UNESP/ Marília - SP e somente teve início após a sua aprovação (CEP/FFC-CM № 3090/2008).

A análise estatística dos dados da ELASI, nas comparações entre os grupos, foi feita pela prova $U$ de Mann-Whitney e os resultados foram considerados estatisticamente significantes quando $p<0,05$.

Após o preenchimento dos questionários, foi promovida a categorização das respostas obtidas. Foram selecionados, para análise e discussão, principalmente as categorias que obtiveram maior freqüência de respostas, pois retratam de maneira geral os conhecimentos e opiniões desses 
profissionais. Para as categorias nas quais era importante verificar o comportamento dos grupos, a fim de saber se existiam diferenças em decorrência da experiência dos professores com alunos com perda auditiva ou da série em que os alunos estavam cursando, foi aplicado o Teste do Quiquadrado $\left(\mathrm{c}^{2}\right)$ ou Prova Exata de Fisher. Os grupos foram considerados diferentes do ponto de vista estatístico, quando $p<0,05$.

\section{RESULTADOS}

Os resultados apurados na avaliação das atitudes em relação à inclusão encontram-se na Tabela 1 , com os escores medianos separados por itens ideológicos e operacionais.

Os escores totais da ELASI foram desmembrados em escores dos itens ideológicos e os dos itens operacionais para fins de análise. A análise comparativa realizada com o auxílio da prova de Mann-Whitney indicou que os escores obtidos por participantes do grupo 1 não diferem significantemente dos do grupo 2, nem para os itens ideológicos $(p=0,2080)$ nem para os itens operacionais $(p=0,1151)$ na ELASI forma $A$. Procedendo aos mesmos cálculos com os escores da ELASI forma $B$, foram obtidos resultados que indicam não haver diferença significante entre os escores do grupo $1 \mathrm{e}$ os do grupo 2, nem nos itens ideológicos ( $p=0,6005)$ nem nos itens operacionais $(p=0,2624)$. Esse resultado sugere que para professores de $1^{\text {a }}$ a $4^{\text {a }}$ séries, a experiência com alunos surdos não afetou a aceitação do processo de inclusão.

A comparação dos resultados do grupo 3 com os do grupo 4 revelou que não houve diferença significante nos itens ideológicos $(p=0,4079)$. Já em relação aos itens operacionais, a comparação por meio da prova de Mann-Whitney mostrou haver diferença estatisticamente significante entre os escores do grupo 3 e os do grupo 4 ( $p=0,0274)$. Esse resultado sugere que entre os professores que ministravam aulas no Ensino Fundamental, da $5^{\underline{a}}$ a $8^{\mathrm{a}}$ séries, a experiência com aluno deficiente pareceu afetar positivamente as suas atitudes sociais em relação à inclusão.

Com relação aos conhecimentos dos professores a respeito da deficiência auditiva, as respostas do questionário foram organizadas em dois temas.

No primeiro tema, foi verificado o conhecimento dos professores a respeito da deficiência auditiva, dos diferentes graus de perda auditiva, recursos tecnológicos para o aproveitamento da audição residual e a distância para se efetivar a comunicação.

Todos os professores afirmaram que a perda auditiva pode ocorrer em diferentes graus, mas nem todos justificaram a sua resposta, $65,4 \%$ dos professores do grupo 1 e $84,2 \%$ dos professores do grupo 2 justificaram a sua resposta. Considerando que a ocorrência da justificativa pode sugerir um conhecimento maior sobre o assunto, foram comparados os professores de $1^{\underline{a}}$ a $4^{\mathrm{a}}$ séries que nunca tiveram contato com alunos deficientes auditivos com os que já tiveram essa experiência. Foi encontrado o $c^{2}$ no valor de 1,453 , com um grau de liberdade e probabilidade de 0,2850 . Portanto, embora haja uma porcentagem maior de professores com experiência que justificaram a resposta, essa diferença não chega a ser estatisticamente significante.

Entre os professores de $5^{\text {a }}$ a $8^{\text {a }}$ séries, $28,6 \%$ dos professores do grupo 3 e $40 \%$ dos professores do grupo 4 justificaram a sua resposta. Comparando esses dois grupos, por meio da prova exata de Fisher, obteve-se a probabilidade 1, isto é, os grupos são estatisticamente equivalentes.

Deve-se destacar que os professores da $1^{\underline{a}}$ a $4^{\underline{a}}$ séries mantêm contato diário com seus alunos, ao passo que os da $5^{\underline{a}}$ a $8^{\underline{a}}$ séries mantêm contato apenas durante as aulas da respectiva disciplina. Tal experiência diferenciada de contato pode influir no conhecimento que possam ter acerca da deficiência auditiva de seus alunos. Assim, foram comparadas

Tabela 1 - Mediana dos escores obtidos da ELASI em relação aos itens ideológicos e operacionais

\begin{tabular}{|c|c|c|c|c|c|}
\hline & \multirow[b]{2}{*}{ Itens } & \multicolumn{2}{|c|}{ 1a a 4 $4^{a}$ séries } & \multicolumn{2}{|c|}{$5^{\mathrm{a}}$ a $8^{\mathrm{a}}$ séries } \\
\hline & & $\begin{array}{c}\text { Grupo } 1 \\
\text { Sem Exp. } \\
(n=26)\end{array}$ & $\begin{array}{c}\text { Grupo } 2 \\
\text { Com Exp. } \\
(n=19)\end{array}$ & $\begin{array}{c}\text { Grupo } 3^{*} \\
\text { Sem Exp. } \\
(n=7)\end{array}$ & $\begin{array}{c}\text { Grupo } 4^{*} \\
\text { Com Exp. } \\
(n=20)\end{array}$ \\
\hline \multirow{2}{*}{ Forma A } & Ideológicos & 95 & 97 & 95 & 95 \\
\hline & Operacionais & 30 & 34 & 26 & 34 \\
\hline \multirow{2}{*}{ Forma B } & Ideológicos & 82 & 77 & ----- & ----- \\
\hline & Operacionais & 42 & 38 & ----- & ----- \\
\hline
\end{tabular}

* Os grupos 3 e 4 responderam apenas a forma A da ELASI. 
as respostas do grupo 2 com as do grupo 4, ambos constituídos por professores que têm experiência com alunos deficientes auditivos. Foi obtido o $c^{2}$ no valor de 6,287, com um grau de liberdade e probabilidade de 0,0061 . Pode-se concluir que significantemente mais professores do grupo 2 justificaram a sua resposta, comparativamente ao grupo 4 .

Parte dos professores (G2 $=52,4 \%$ e $G 4=80 \%$ ), com experiência com aluno deficiente auditivo, não sabia informar o grau da perda auditiva dos seus alunos.

A maioria dos professores demonstrou não conhecer os recursos utilizados para possibilitar o acesso ao som às pessoas com perda auditiva (Tabela 2). As respostas consideradas pertinentes foram aquelas que citavam algum tipo de descrição ou utilização dos recursos. Essa categoria foi obtida somente entre os professores de $1^{\underline{a}}$ a $4^{\underline{a}}$ séries.

As respostas obtidas dos professores em relação ao AASI (grupos 1 e 2) foram analisadas com a prova exata de Fisher, obtendo-se o valor da probabilidade de 0,2528. Assim, pode-se concluir que a experiência com alunos com perda auditiva não favoreceu maior conhecimento em relação a esse recurso de amplificação sonora. Dentre os professores com experiência com alunos com perda auditiva, há estatisticamente mais professores da $1^{\underline{a}}$ a $4^{a}$ séries que forneceram respostas pertinentes que os da $5^{\underline{a}}$ a $8^{\text {a }}$ séries, pois, nessa comparação, por meio da prova exata de Fisher, foi obtida a probabilidade de 0,0202.

Com respeito à necessidade de se falar mais próximo do aluno deficiente auditivo não foi observado diferenças expressivas entre os grupos de professores. Apontam essa necessidade 42,3\% dos professores do $\mathrm{G} 1,57,8 \%$ dos professores do G2, $57,1 \%$ dos professores do G3 e $40 \%$ dos professores do G4.

A maior parte dos professores com experiência com alunos com perda auditiva informou que utilizava a comunicação oral na sala de aula. As principais ocorrências de categorias de respostas, nesse tópico, estavam relacionadas a mudar a estratégia comunicação (G2=68,4\% e G4=65\%) e utilizar gestos de apoio (G2=21,1\% e G4=25\%). Os professores que não se comunicavam oralmente com os alunos deficientes auditivos informaram que solicitam auxílio para outras pessoas (professores ou alunos que conheçam a língua de sinais) ou utilizam à comunicação escrita como apoio (G2=10,5\% e G4=10\%). Os professores, no entanto, não apresentam nas respostas informações que indicavam que as estratégias utilizadas eram adequadas e eficientes para os alunos com perda auditiva, com os quais atuavam no ensino regular.

Esse aspecto também é evidenciado quando questionados a respeito de sugestões para auxiliar a comunicação com o aluno com perda auditiva, as respostas fornecidas por todos os professores foram bastante diversificadas (Tabela 3).

Outro resultado destacado, nesse estudo, é que grande parte dos professores acredita que o ensino especial seja o mais adequado para o aluno deficiente auditivo ( $\mathrm{G} 1=76,9 \%, \mathrm{G} 2=52,6 \%, \mathrm{G} 3=71,4 \%$ e $\mathrm{G} 4=30 \%$ ).

\section{DISCUSSÃO}

Com relação às atitudes sociais acerca de inclusão, pelo fato de o fundamento da educação inclusiva apoiar-se na proposta da igualdade de direitos ${ }^{19} \mathrm{e}$ de esse argumento ser algo praticamente incontestável, não causa estranheza que os professores ofereçam respostas semelhantes para os itens ideológicos da escala, independentemente de terem ou não experiência com alunos com perda auditiva.

Do ponto de vista operacional, os professores de $5^{\mathrm{a}}$ a $8^{\mathrm{a}}$ séries com experiência com alunos com perda auditiva parecem vislumbrar maiores pos-

Tabela 2 - Frequência (\%) de categorias encontradas sobre o conhecimento dos professores acerca dos recursos tecnológicos existentes para facilitar a comunicação com o aluno com perda auditiva

\begin{tabular}{|c|c|c|c|c|c|}
\hline \multirow[b]{2}{*}{ Recursos } & \multirow[b]{2}{*}{ Categorias } & \multicolumn{2}{|c|}{$1^{a}$ a $4^{a}$ séries } & \multicolumn{2}{|c|}{$5^{\mathrm{a}}$ a $8^{\mathrm{a}}$ séries } \\
\hline & & $\begin{array}{c}\text { Grupo } 1 \\
\text { Sem Exp. } \\
(n=26)\end{array}$ & $\begin{array}{c}\text { Grupo } 2 \\
\text { Com Exp. } \\
(n=19)\end{array}$ & $\begin{array}{c}\text { Grupo } 3 \\
\text { Sem Exp. } \\
(n=7)\end{array}$ & $\begin{array}{c}\text { Grupo } 4 \\
\text { Com Exp. } \\
(n=20)\end{array}$ \\
\hline \multirow{2}{*}{ AASI } & Nenhum & $23(88,5 \%)$ & $14(73,7 \%)$ & $7(100 \%)$ & $20(100 \%)$ \\
\hline & Pertinente & $3(11,5 \%)$ & $5(26,3 \%)$ & $0(0 \%)$ & $0(0 \%)$ \\
\hline FM & Nenhum & $26(100 \%)$ & $19(100 \%)$ & $7(100 \%)$ & $20(100 \%)$ \\
\hline \multirow{2}{*}{ IC } & Nenhum & $25(96,2 \%)$ & $19(100 \%)$ & 7 (100\%) & $20(100 \%)$ \\
\hline & Pertinente & $1(3,8)$ & $0(0 \%)$ & $0(\%)$ & $0(\%)$ \\
\hline
\end{tabular}


Tabela 3 - Frequência (\%) de ocorrência de categorias de respostas relativas a estratégias de comunicação

\begin{tabular}{|c|c|c|c|c|}
\hline \multicolumn{5}{|c|}{ Categorias relacionadas a aspectos que auxiliam a comunicação } \\
\hline & \multicolumn{2}{|c|}{ 1a a $4^{a}$ séries } & \multicolumn{2}{|c|}{$5^{\mathrm{a}}$ a $8^{\mathrm{a}}$ séries } \\
\hline & $\begin{array}{c}\text { Grupo } 1 \\
\text { Sem Exp. } \\
(n=26)\end{array}$ & $\begin{array}{c}\text { Grupo } 2 \\
\text { Com Exp. } \\
(n=19)\end{array}$ & $\begin{array}{c}\text { Grupo } 3 \\
\text { Sem Exp. } \\
(n=7)\end{array}$ & $\begin{array}{c}\text { Grupo } 4 \\
\text { Com Exp. } \\
(n=20)\end{array}$ \\
\hline Gestos de apoio & $9(34,6 \%)$ & $4(21,0 \%)$ & $1(14,3)$ & $4(20 \%)$ \\
\hline $\begin{array}{l}\text { Recursos didáticos } \\
\text { e monitoria }\end{array}$ & $4(15,4 \%)$ & $6(31,6 \%)$ & $0(0 \%)$ & $4(20 \%)$ \\
\hline $\begin{array}{c}\text { Equipe } \\
\text { especializada }\end{array}$ & $4(15,4 \%)$ & $6(31,5)$ & $0(0 \%)$ & $2(15 \%)$ \\
\hline $\begin{array}{c}\text { Bom } \\
\text { relacionamento }\end{array}$ & $1(3,8 \%)$ & $1(5,3 \%)$ & $2(28,6 \%)$ & $0(0 \%)$ \\
\hline $\begin{array}{l}\text { Formação } \\
\text { específica }\end{array}$ & $8(30,8 \%)$ & $1(5,3 \%)$ & $0(0 \%)$ & $4(20 \%)$ \\
\hline Não sei & $0(0 \%)$ & $1(5,3 \%)$ & $4(57,1 \%)$ & $3(15 \%)$ \\
\hline Outras & $0(0 \%)$ & $0(0 \%)$ & $0(0 \%)$ & $2(10 \%)$ \\
\hline
\end{tabular}

sibilidades de se efetivar a proposta da inclusão, demonstrando atitudes sociais mais favoráveis, comparativamente a seus pares sem experiência com alunos deficientes auditivos. Talvez isso esteja associado ao pouco contato que esses professores possuem com os alunos deficientes auditivos. Pode-se atentar também a hipótese de que as características dos alunos deficientes auditivos que estão frequentando essas séries, diferentemente das características dos alunos deficientes auditivos que frequentam as séries iniciais podem sugerir aos professores, mesmo com pouco contato, a possibilidade de plena escolarização.

O estudo das atitudes é ressaltado em diversos trabalhos ${ }^{9,12-14}$ como essencial para a viabilização de alterações no ambiente social, de modo a favorecer a prática de ensinar os alunos com necessidades educacionais especiais juntamente com os demais, que não apresentam essas necessidades.

Quanto ao conhecimento dos professores sobre aspectos inerentes a deficiência auditiva destacase o fato de o grupo de professores com experiência não apresentar respostas substancialmente diferentes, na maioria das questões. Evidentemente, é esperado que os professores do ensino regular não tenham conhecimentos específicos em relação à deficiência auditiva. Isso não significa, entretanto, que, na educação inclusiva, esses professores não necessitem adotar condutas adequadas para cada aluno com perda auditiva, mediante avaliação de especialistas ${ }^{20}$.

As diferenças encontradas entre as respostas obtidas dos questionários dos professores com experiência, que trabalham nas séries iniciais (grupo 2) e finais (grupo 4) do ensino fundamental, podem estar ligadas ao fato de os professores de $1^{\text {a }}$ a $4^{a}$ séries permanecerem mais tempo com os seus alunos e, dessa forma, poderem observar melhor esses alunos.

Conhecer o grau da perda auditiva e principalmente as consequências relacionadas às habilidades auditivas e comunicativas é uma importante condição para o desenvolvimento acadêmico de um aluno com perda auditiva.

Todas as pessoas que trabalham com alunos com perda auditiva deveriam conhecer o potencial e as limitações dos recursos de amplificação. Embora os conhecimentos sobre esses recursos sejam importantes, no presente trabalho observa-se que os professores apresentam poucos conhecimentos sobre os recursos tecnológicos que auxiliam o desenvolvimento de alunos com perda auditiva.

As estratégias de comunicação utilizadas pelo professor de um aluno deficiente auditivo, usuário de aparelho de amplificação sonora individual ou de implante coclear, que possui possibilidades de percepção dos sons da fala, precisam ser diferentes daquelas utilizadas com alunos que se apropriam da Língua Brasileira de Sinais. Os professores participantes deste estudo não demonstraram conhecer tais estratégias, fato este que certamente dificulta a construção de um ambiente social acolhedor na sala de aula, para alunos deficientes auditivos, o qual poderia auxiliar o desenvolvimento de linguagem e de aprendizagem desses alunos. 
Após analise critica do estudo realizado cabe comentar que :

- devem ser tomadas providências no período pré-escolar para melhorar desenvolvimento das crianças deficientes auditivas. Dentre estas, estão o desenvolvimento da comunicação oral ou gestual, as quais irão influenciar sobremaneira o desenvolvimento acadêmico.

- Investimentos no diagnóstico precoce, recursos tecnológicos de dispositivos que possibilite o acesso aos sons da fala e acompanhamento sistemático das famílias, também são aspectos que irão influenciar a viabilização da participação do aluno com perda auditiva no contexto escolar.

\section{CONCLUSÕES}

Os dados provenientes de instrumentos diferentes utilizados se complementam e sugerem que os professores são ideologicamente favoráveis à inclusão, mas não têm conhecimentos suficientes para operacionalizar a proposta de educação inclusiva. Torna-se evidente a importância da realização de programas de apoio aos professores e programas que atendam as necessidades de crianças com perda auditiva, nos primeiros anos de vida, em momentos que antecedem o início do período escolar. Essas medidas poderão refletir pontualmente na relação professor/aluno e no desenvolvimento acadêmico dessas crianças.

\begin{abstract}
Purpose: to check teachers' knowledge about hearing loss, their opinion about the students with this kind of sensorial privation and to know about their attitudes towards the inclusion proposal. Methods: four groups of teachers from primary and secondary school, with and without experience with hearing loss students, took part in this research. The instruments utilized on this data were Lickert Scale of Attitudes toward Inclusion (ELASI) and a questionnaire, comparisons between different groups, by means of proper statistical tools, whenever the data features recommended it. Results: the primary school teachers, with and without experience on hearing loss students, showed similar responses related to social attitudes about inclusion, both in under the ideological and operational dimension. The secondary school teachers, with and without experience, showed similar responses on the ideological items, however, they disagreed on the operational items. Related to the knowledge about hearing loss aspects, when compared with the group without experience and all groups emphasized the communicative aspects. Conclusion: the analysis revealed that different data collection procedures produced complementary data and suggested that teachers were ideologically favorable to inclusion, but they had not enough knowledge to make it feasible.
\end{abstract}

KEYWORDS: Education; Rehabilitation of Hearing Impaired; Deafness; Hearing Loss

\section{REFERÊNCIAS}

1. Willstedt-Svensson U, Löfqvist $A$, Almqvist B, Sahlén B. Is age at implant the only factor that counts? The influence of working memory on lexical and grammatical development in children with cochlear implants. Int J Audiol. 2004; 43(9):506-15. 2. Boothroyd A. Hearing aids, cochlear implants and profoundly deaf children. In: Owens E, Kessler DK. Cochlear implants in young deaf children. San Francisco: College-Hill Publication; 1989. p. 81-99.

3. Harrison RV, Gordon KA, Mount RJ. Is there a critical period for cochlear implantation in congenitally deaf children? Analyses of hearing and speech perception performance after implantation. Develop Psychobiol. 2005; 46(3):252-61.
4. Tomblin JB, Baker BA, Spencer LJ, Zhang X, Gantz BJ. The effect of age at cochlear implant initial stimulation on expressive language growth in infants and toddlers. J Speech Lang Hear Res. 2005; 48(4):853-67.

5. Pádua FGM, Marone S, Bento RF, Carvallo RMM, Durante AS, Soares JC, et al. Triagem auditiva neonatal: um desafio para sua implantação. Arq Int Otorrinolaringol. 2005; 9(3):328-34.

6. Barreira-Nielsen C, Futuro Neto HA, Gattaz G. Processo de implantação de programa de saúde auditiva em duas maternidades públicas. Rev Soc. Bras. Fonoaudiol. 2007; 12(2):99-105.

7. Félix A. O papel da interação no processo de ensino-aprendizagem de português para alunos surdos em uma escola inclusiva. Trab. Linguist. 
Aplic. 2009; 48(1):119-31. http://dx.doi.org/10.1590/ S0103-18132009000100009

8. Marlatt EA. Measuring practical knowledge among prospective and current teachers of deaf and hard of hearing students. Am Ann Deaf. 2001; 146(4):331-47.

9. Lanier NJ, Lanier WL. The effects of experience on teachers' attitudes toward incorporating special students into the regular classroom. Education. 1996; 117(2):234-7.

10. Luckner JL. Mainstreaming hearing-impaired students: perceptions of regular educators. Lang Speech Hear Serv Schools. 1991; 22(1):302-7.

11. Martinez CMS, Pamplin RCO, Oishi J. Professores de crianças com necessidades educacionais especiais: o suporte informacional no cotidiano da escola que busca a inclusão. Rev. Bras. Educ. Esp. 2005; 11(1):67-80.

12. Monahan RG, Marino SB, Miller R. Teacher attitudes toward inclusion: implications for teacher education in schools. Education. 1996; 117(2):316-21.

13. Barker KS, Shoho AR, Van Reusen AK. High school teacher attitudes toward inclusion (statistical data included). High School J. 2000; 84(2):7-20.

14. Omote S, Oliveira AAS, Baleotti LR, Martins SESO. Mudança de atitudes sociais em relação à inclusão. Padéia. 2005; 15(32):387-98.
15. Silva SC, Aranha MSF. Interação entre professora e alunos em salas de aula com proposta pedagógica de educação inclusiva. Rev. Bras. Educ. Esp. 2005; 11(3):373-94.

16. Buffa MJMB. A inclusão da criança deficiente auditiva no ensino regular: uma visão do professor de classe comum. [dissertação] Bauru (SP): Universidade de São Paulo; 2002. 119f.

17. Sacaloski M. Inserção do aluno deficiente auditivo no ensino regular: a comparação entre o desempenho dos alunos ouvintes e deficientes auditivos e a visão dos pais, professores e alunos. [tese] São Paulo (SP): Universidade Federal de São Paulo; 2001. 150f.

18. Omote S, Delgado EMC, Fabron EMG, Moraes ACPF. Estudo da fidedignidade de uma escala de mensuração de atitudes sociais em relação à inclusão. In: Anais do Simpósio em Filosofia e Ciência. 2003, Marília. Marília: UNESP; 2003c. [CD-ROM].

19. Karagiannis A, Stainback W, Stainback S. Fundamentos do ensino inclusivo. In: Stainback $S$, Stainback W. Inclusão: um guia para educadores. Porto Alegre: ArtMed; 1999. p. 21-34.

20. Angelides $P$, Aravi $C$. The development of inclusive practices as a result of the process of integrating deaf/hard of hearing students. Eur $\mathrm{J}$ Spec Needs Educ. 2007; 22(1):63-74.

DOI: 10.1590/S1516-18462010005000024

RECEBIDO EM: 23/06/2009

ACEITO EM: 04/12/2009

Endereço para Correspondência

Eliane Maria Carrit Delgado-Pinheiro

Rua José da Silva Matos, 350 -

Condomínio Pedra Verde

Marília - SP

CEP: $17516-540$

E-mail: elia@ terra.com.br 